\title{
BMJ Open PEBBLES study protocol: a randomised controlled trial to prevent atopic dermatitis, food allergy and sensitisation in infants with a family history of allergic disease using a skin barrier improvement strategy
}

\author{
Adrian Lowe, ${ }^{01,2}$ John Su, ${ }^{2,3,4,5}$ Mimi Tang, ${ }^{2,3,5}$ Caroline J Lodge, ${ }^{1,2}$ \\ Melanie Matheson, ${ }^{1}$ Katrina J Allen, ${ }^{2,3}$ George Varigos, ${ }^{6}$ Arun Sasi, ${ }^{7}$ \\ Noel Cranswick, ${ }^{2,5}$ Simone Hamilton, ${ }^{1,2}$ Colin F Robertson, ${ }^{2,5}$ Jennie Hui, ${ }^{8}$ \\ Michael Abramson, ${ }^{9}$ Shaie O'Brien, ${ }^{1,2}$ Shyamali Dharmage ${ }^{1,2}$
}

To cite: Lowe A, Su J, Tang M, et al. PEBBLES study protocol: a randomised controlled trial to prevent atopic dermatitis, food allergy and sensitisation in infants with a family history of allergic disease using a skin barrier improvement strategy. BMJ Open 2019;9:e024594. doi:10.1136/ bmjopen-2018-024594

\section{- Prepublication history for} this paper are available online. To view these files, please visit the journal online (http://dx.doi org/10.1136/bmjopen-2018024594).

Received 4 June 2018

Revised 21 November 2018

Accepted 11 January 2019

A Check for updates

(C) Author(s) (or their employer(s)) 2019. Re-use permitted under CC BY-NC. No commercial re-use. See rights and permissions. Published by BMJ.

For numbered affiliations see end of article.

Correspondence to

Adrian Lowe;

lowea@unimelb.edu.au

\section{ABSTRACT}

Introduction The skin is an important barrier against environmental allergens, but infants have relatively impaired skin barrier function. There is evidence that impaired skin barrier function increases the risk of allergic sensitisation, atopic dermatitis (AD) and food allergy. We hypothesise that regular prophylactic use of emollients, particularly those that are designed to improve skin barrier structure and function, will help prevent these conditions. With the aim of determining if application of a ceramide-dominant emollient two times per day reduces the risk of $A D$ and food allergy, we have commenced a multicentre phase III, outcome assessor blinded, randomised controlled trial of this emollient applied from birth to 6 months.

Methods and analysis Infants $(n=760)$ with a family history of allergic disease will be recruited from maternity hospitals in Melbourne. The primary outcomes are as follows: the presence of $A D$, assessed using the UK Working Party criteria, and food allergy using food challenge, in the first 12 months of life as assessed by a blinded study outcome assessor. Secondary outcomes are as follows: food sensitisation (skin prick test), skin barrier function, $A D$ severity, the presence of new onset $A D$ after treatment cessation (between 6 and 12 months) and the presence of parent reported AD/eczema. Recruitment commenced in March 2018.

Ethics and dissemination The PEBBLES Study is approved by the Human Research Ethics Committees of the Royal Children's Hospital (RCH) (\#37090A) and the Mercy Hospital for Women (2018-008). Parents or guardians will provide written informed consent. Outcomes will be disseminated through peer-reviewed publications and presented at scientific conferences.

Trial registration numbers ACTRN12617001380381 and NCT03667651.
Strengths and limitations of this study

- This large randomised trial will use robust methods to investigate the impact of application of a ceramide-dominant emollient two times per day on atopic dermatitis and food allergy.

- Using this emollient may maximise the effectiveness of this intervention, as ceramides are important in the formation of the skin barrier and neonatal skin is relatively deficient in ceramides.

- Parent adherence to the intervention may vary and wane over time and this may reduce the treatment effect.

- Participants in the control group may use other emollients, potentially leading to underestimating the efficacy of the intervention.

- Recruiting only children with a family history of allergic disease may limit the generalisability of the results to the broader population.

\section{INTRODUCTION}

Allergic diseases are common, cause substantial burden of disease and their causes remain largely unknown. Except for early introduction of peanut and egg to prevent food allergies to these specific allergens, ${ }^{1}$ there are limited proven primary prevention strategies available. Given the accumulating evidence on the link between infant atopic dermatitis (AD) and increased risk of developing food allergy, ${ }^{2}$ asthma ${ }^{3}$ and allergic rhinitis, there is increasing interest in investigating whether improving skin barrier function could prevent subsequent childhood allergies. We have been actively investigating the role of $\mathrm{AD}$ in the development of sensitisation, food 


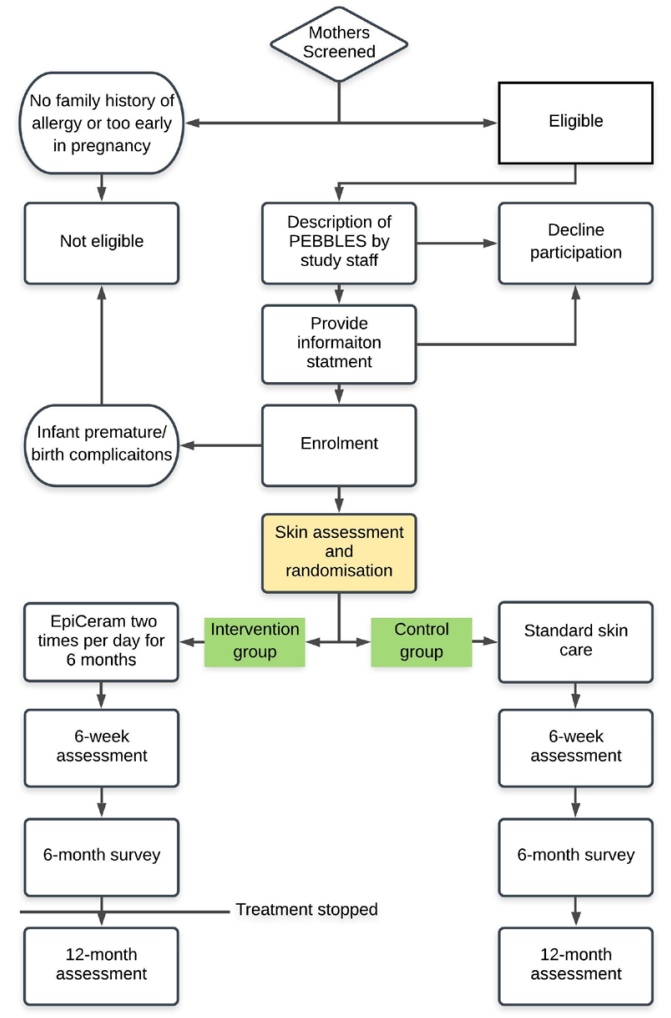

Figure 1 Study flow diagram.

allergy and asthma both in childhood ${ }^{2-5}$ and into adulthood. ${ }^{67}$ Despite AD typically being the first sign of allergy, there is no definitive proof that $\mathrm{AD}$ causes food allergy, hay fever or asthma, as these conditions may be associated due to shared genetic and environment causes. Twin and sibling studies highlight the importance of genetic causes of these conditions, although the link between $\mathrm{AD}$ and allergic airways disease is independent of shared early-life environmental factors. ${ }^{8}$

Biological plausibility exists for a causal link between $\mathrm{AD}$ and other allergies. Preventing infants from developing $\mathrm{AD}$ may halt progression to the next step in the atopic disease, as it is possible that sensitisation to allergens occurs via damaged skin associated with $\mathrm{AD}$, which then increases the risk of these other conditions. ${ }^{9}$ There are a range of data that support this concept. Skin is an essential barrier to the external environment, and infants have relatively poor skin barrier function. ${ }^{10}$ The skin barrier is impaired in $\mathrm{AD}$ as shown by higher levels of transepidermal water loss (TEWL) through both affected and asymptomatic skin. ${ }^{11}$ Reduced skin barrier function in the first week of life is associated with an increased risk of $\mathrm{AD}$ by 12 months of age. ${ }^{12}$

To date, three small clinical trials (sample sizes ranging from 80 to 124) have demonstrated that daily application of an emollient to the skin of infants can reduce the incidence of $\mathrm{AD}$ by approximately $50 \% .^{13-15}$ These studies establish the proof of concept for $\mathrm{AD}$ prevention using emollients to improve barrier function of skin. These studies have used emollients as prophylactic treatments from the first weeks of life up to 26 and 32 weeks. It remains to be determined whether (i) there is a sustained reduction in the incidence of $\mathrm{AD}$ once treatment has ceased, (ii) this form of intervention reduces the incidence of food allergy or later asthma and allergic rhinitis and (iii) such treatments are effective in both those with and without filaggrin (FLG) null mutations. Larger randomised controlled trials, powered to demonstrate the long-term effects of this form of treatment on the incidence of $\mathrm{AD}$ beyond the treatment period, and measurement of food allergy outcomes, are urgently needed. A number of such trials are already underway to address these gaps in knowledge. ${ }^{16}$

Two of the trials to date have used standard emollients. ${ }^{1415}$ These have included 2e Douhet emulsion in one study ${ }^{15}$ or a range of products selected by the parents (sunflower seed oil, double-base gel, liquid paraffin in white paraffin, Cetaphil cream or Aquaphor) in the other. ${ }^{14}$ In contrast, in our own PEBBLES (Prevention of Eczema $\underline{B}$ y a Barrier Lipid Equilibrium $\underline{\text { Strategy) pilot }}$ study, we have used a ceramide-dominant emollient. ${ }^{13}$ The poorer barrier function of the skin of infants is, at least in part, due to low concentrations of ceramides in the stratum corneum, with ceramide concentrations increasing in the weeks following birth. ${ }^{17}$ Diseased skin, including eczematous skin, is also characterised by decreased ceramide levels. ${ }^{11}$

EpiCeram has been formulated to have the optimum ratio $(3: 1: 1)$ of ceramides, cholesterol and fatty acids for skin barrier restoration. ${ }^{18}$ It contains the major fatty components of human skin at physiological concentrations, and it has been shown to greatly accelerate the restoration of the skin barrier function $(>90 \%$ at 4 hours following application). In addition, EpiCeram has a slightly acidic $\mathrm{pH}(5.0)$, similar to mature skin, which aids the production and secretion of ceramides by the skin. ${ }^{19}$ Notably, newborn vernix caseosa is also rich in cholesterol, fatty acids and ceramides. ${ }^{17}$ EpiCeram has a similar impact on the symptoms of $\mathrm{AD}$ as a moderate strength topical corticosteroid (TCS) cream, ${ }^{20}$ without potential TCS side effects. ${ }^{21}$ It has been well tolerated as a prophylactic treatment in infants in both of our previous studies. ${ }^{1322}$ In a per protocol analysis, we have previously observed a promising reduction in food sensitisation at 12 months in those children whose parents applied EpiCeram on average at least 5 days per week (0\% vs $19 \%, \mathrm{p}=0.04) .{ }^{13}$ Due to its potential benefits, we have chosen to use EpiCeram rather than a standard emollient as our intervention in our trial described here.

Given the above gaps in knowledge, we have designed the PEBBLES study, a randomised, parallel arm, singleblinded, trial to test if routine use of a ceramide-dominant emollient for the first 6 months of life prevents the development of $\mathrm{AD}$, including beyond the active treatment period, or reduces the prevalence of food allergy and allergic sensitisation. This study follows on from our published pilot study. ${ }^{13}$ The results of this trial will help 
clarify the role of the skin barrier as a route of allergic sensitisation and subsequent allergic disease.

\section{OBJECTIVES}

To determine if application of a ceramide-dominant emollient two times per day reduces the risk of $\mathrm{AD}$ and food allergy by 12 months of age, when compared with standard skin management. We also aim to determine if the intervention reduces the risk of allergic sensitisation (as measured by skin prick tests), improves infant skin barrier function, causes any adverse effects in infants and influences infant skin microbial colonisation or skin lipid profile.

\section{METHODS}

\section{Design}

This is a phase III, single-blind (outcome assessment is blinded), randomised controlled multicentre trial of the effect of EpiCeram emollient on the primary outcomes of incidence of $\mathrm{AD}$ and food allergy in high risk infants.

\section{Setting and participants}

Treatment will be from birth until 6 months of age, with a 6-week, 6-month (26 weeks) and 12-month (52 weeks) follow-up. Infants with a family history of allergic disease (biological parent or half/full sibling) will be recruited from maternity hospitals in Melbourne, Australia. The study diagram is depicted in figure 1 . We aim to recruit 760 infants (380 per arm). Recruitment will occur at the Royal Women's Hospital, Frances Perry House, Mercy Hospital for Women and the Murdoch Children's Research Institute.

\section{Inclusion and exclusion criteria \\ Inclusion criteria}

Only full-term infants with a family history (biological parent or full/half sibling) of allergic disease will be eligible to ensure a high rate of $\mathrm{AD}$ and food allergy. Infants will be eligible to participate if at least one immediate family member (biological mother, father or older sibling) has a self-reported history of at least one of asthma, eczema/AD, hay fever/allergic rhinitis or food allergy. Infants will be enrolled up to 3 weeks of age. Pregnant mothers may also prenatally enrol their child into the study from 28 weeks' gestation, but randomisation will only occur post birth.

\section{Exclusion criteria}

Infants with the following will be excluded: a parent who has a known hypersensitivity to any of the ingredients of EpiCeram, multiple births, born premature $(<36$ weeks), major birth or early life medical complications; parents who do not have sufficient English language skills to answer questions and those who would be unable to comply with all protocol required visits and procedures.

\section{Randomisation}

A computer-generated random 1:1 allocation list in blocks of variable length (4-8) will be developed and loaded onto the study's REDCap database by the lead trial statistician (Lyle Gurrin). The list will be stratified based on study recruitment site and number of family members with allergic disease ( 1 vs $>1)$, to ensure balance between the groups on these potentially prognostic factors. Consenting participants meeting inclusion criteria will be randomised and allocated study numbers. At all times, the allocation list will be concealed from the study coordinator and other investigators, who manage participant recruitment and follow-up.

\section{Intervention and control conditions}

Infants will be randomly assigned to receive either standard skin care advice (control group) or two times per day treatment with EpiCeram (intervention group). EpiCeram has been approved for use by patients with $\mathrm{AD}$ or eczema by the Food and Drug Administration (FDA) in the USA but does not yet have Australian Therapeutic Goods Administration (TGA) approval and is not currently available in Australia. Parents will be instructed to apply approximately $6 \mathrm{~g}$ of EpiCeram per application two times per day from birth until the infant is 6 months of age. Parents of children in the control group will be managed as per existing practice and will not be given any emollients. For ethical reasons, parents of children in the control group will not be told to withhold skin care from their infant and information relating to use of emollients will be collected from all participants.

\section{Compliance}

A weekly diary will be completed online by parents who will document the frequency of EpiCeram application and use of any other creams. Parents will also be asked to return all tubes of cream (used and unused) at all follow-up appointments, so the study team can weigh any remaining cream. Our pilot study found that $76 \%$ of parents in the intervention group applied the cream on 5 or more days per week. ${ }^{13}$ Use of other emollients and skin care products will also be recorded at the same time.

\section{Outcomes}

Primary

- The presence of AD in the first 12 months of life assessed using the UK Working Party criteria ${ }^{23}$ and/or visible $\mathrm{AD}$ at the time of examinations.

- Food allergy, based on skin prick tests, history of reactions and food challenge at 12 months. ${ }^{24}$

\section{Secondary}

- Adverse reaction to EpiCeram.

- Skin barrier function as assessed by TEWL at 6 weeks and 12 months.

- Food sensitisation (positive skin prick test) at 12 months of age.

- Presence of observed $\mathrm{AD}$ that first presents from 6 to 12 months (incident after intervention period). 
Table 1 Timing of study measures

\begin{tabular}{|c|c|c|c|c|c|c|}
\hline Study period & Screening & Baseline & $\begin{array}{l}\text { Telephone/ } \\
\text { email contact-1 }\end{array}$ & Follow-up-1 & Follow-up-2 & Follow-up-3 \\
\hline Visit number & Visit 0 & Visit 1 & & Visit 2 & Visit 3 & Visit 4 \\
\hline Week & $\begin{array}{l}28 \text { weeks } \\
\text { gestation } \\
-3 \text { weeks }\end{array}$ & 1 day-3 weeks & 4 weeks & 6-12 weeks & $\begin{array}{l}\text { 6-9 months } \\
\text { (26-39 weeks) }\end{array}$ & $\begin{array}{l}\text { 12-24 months } \\
\text { (52-104 weeks) }\end{array}$ \\
\hline Informed consent & & $x$ & & & & \\
\hline Demographic & & $x$ & & & & \\
\hline $\begin{array}{l}\text { Family history/home } \\
\text { environment }\end{array}$ & & $x$ & & & & \\
\hline Skin assessment & & $x$ & & $x$ & & $x$ \\
\hline Randomisation & & $x$ & & & & \\
\hline Study cream dispensing & & $x$ & & $x$ & & \\
\hline Participant survey & & $x$ & & $x$ & $\mathrm{x}$ & $\mathrm{x}$ \\
\hline Adverse event check & & & $\mathrm{x}$ & $x$ & $x$ & $x$ \\
\hline Diary card & & $x$ & $x$ & $x$ & $x$ & $x$ \\
\hline Compliance check & & & $x$ & $x$ & $x$ & $x$ \\
\hline Skin prick test & & & & & & $x$ \\
\hline Food challenge & & & & & & $x$ \\
\hline \multicolumn{7}{|l|}{ Optional procedures } \\
\hline Skin tape stripping & & $\mathrm{x}$ & & $\mathrm{x}$ & & $\mathrm{x}$ \\
\hline Heel prick test & & $x$ & & $x$ & & $x$ \\
\hline Breast milk sample & & & & $x$ & & \\
\hline Buccal swab & & & & & & $x$ \\
\hline
\end{tabular}

- Presence of probable AD within the first year of life based on parent report of doctor diagnosed AD.

- IgE-associated AD (AD in the context of a positive skin prick test).

- AD severity assessed using the Eczema Area and Severity Index (EASI) score. $^{25}$

\section{Blinding}

Mothers of the infants and the study co-ordinator and recruiter will be aware of the group of allocation. The assessors of the primary outcomes will be blinded to the group of allocation and will have no contact with the study participant beyond measuring of outcomes.

\section{Data collection}

A baseline assessment will be performed when the infant is between 1 day and 3 weeks old (table 1 ). This will entail two surveys, a skin assessment, skin barrier function measurement, tape stripping and an optional heel prick. Subsequent visits, where these tests will be repeated, along with a skin examination for $\mathrm{AD}$, will occur when the infant is 6 weeks and 12 months. The 6 month time-point will be survey only. Breast milk samples will be collected at 6 weeks. At 12 months visit will also entail an optional buccal swab sample for genetic analyses. All infants will receive a skin prick test at 12 months, and infants who are sensitised will be offered a food challenge to determine if they are allergic to those foods.

All participants (in both intervention and control groups) will be provided with the Australasian Society of Clinical Immunology and Allergy's (ASCIA) advice on strategies for allergy prevention (https://www. allergy. org.au / patients / allergy-prevention/asciaguidelines-for-infant-feeding-and-allergy-prevention). This advice, updated in 2016, recommends 'introduction of solid foods around 6 months, but not before 4 months' and that 'introduction of common allergenic foods should not be delayed'. In addition, we will send participants a reminder of this advice at 6 months of age, and monitor introduction of foods into the infant diet as part of the participant surveys.

\section{Data management}

We are using REDCap as our data capture method, either using secure online token-based email links or entered directly on an iPad at baseline. All other data (from skin assessment and skin prick test) will be entered directly into REDCap. No analysis of the data will be conducted until the accuracy of the database has been verified, including assessment for reason for missing data, and that all data are within range. 
All files will be password protected. Participant identifiers will be stored separately from study data, and only be accessible to study staff requiring this information for participant follow-up and interaction.

\section{Primary outcomes}

The presence of $A D$ in the first 12 months of life assessed using a modified version of the UK Working Party criteria for $\mathrm{AD}^{2326}$ and/or visible $\mathrm{AD}$ at the time of examinations. To meet this criteria for $\mathrm{AD}$, a child will need to have history of an itchy skin condition that is either continuous or intermittent and lasting 4 weeks and two or more of (i) history of flexural rash, (ii) a history of dry skin since birth and (iii) visible flexural dermatitis (note-all children will have a family history of allergic disease).

Confirmed diagnosis of food allergy at 12 months. This diagnosis is derived from a combination of allergic sensitisation, reaction history and food challenge, using our previously established definition. ${ }^{24}$ The testing will adhere to ASCIA guidelines.

\section{Secondary outcomes}

Adverse reactions to EpiCeram:

Parents will be asked to immediately contact the study team if they believe that their child has developed an adverse reaction to the EpiCeram treatment. We will instruct parents to cease application of the treatment immediately and wash their infants skin with water. We will organise an assessment with a study dermatologist. Parents will be asked to apply a small amount to the child's forearm on the day prior to the appointment. Reactions to EpiCeram will be confirmed if repeatable symptoms are caused by EpiCeram application.

\section{Surveys}

Questionnaires will be administered at baseline, 6 weeks, 6 months and 12 months. Information to be captured will encompass parental and older sibling history of allergic disease, ${ }^{27}$ ethnicity, home environment, birth details, $\mathrm{AD}$ symptoms, and infant health and medical treatments.

\section{AD/eczema assessment, including severity}

The infant's skin will be evaluated for signs of $\mathrm{AD}$, using $\mathrm{UK}$ Working Party criteria, ${ }^{23}$ at each follow-up and if present it will be assessed for severity using the EASI scale. ${ }^{28}$

\section{Transepidermal water loss}

A closed chambered device (Delfin Technologies Ltd, Kuopio, Finland) will be used at baseline and each follow-up as a non-invasive measure of skin barrier function. Infants' skin will have been exposed and acclimatised to the testing room environment for at least $15 \mathrm{~min}$.

\section{Diary card}

Participants will be asked to complete an online weekly diary to help determine if any adverse events have occurred, treatment compliance for those in the 'cream' group, and to measure other emollient use in both groups.

\section{Skin prick tests}

These will be performed at 12 months on six common allergens (egg white, cow's milk, peanut, dust mite, cat dander and rye grass), along with a negative (saline) and positive (histamine) controls. A Greer prick technique will be used.

\section{Buccal swab collection}

Participants will provide a buccal swab using a Copan nylon flocked swab, and DNA will be extracted from these samples using standardised techniques. FLG null mutations and other mutations related to $\mathrm{AD}$ and food allergy risk $^{29}$ will be examined.

\section{Tape stripping}

Lipids and biome samples will be collected via skin tape stripping at baseline and each follow-up. For lipid sampling, a series of four D-SQUAME Standard adhesive discs will be applied to the same location on forearm skin surface and pressed to apply even pressure for 5-10 s. ${ }^{30}$ To collect skin microbiome samples, a D-SQUAME disc will be repeatedly applied for $2 \mathrm{~min}$ to the same location on the infants (opposing) forearm. ${ }^{31}$ Samples will initially be stored at $-80^{\circ} \mathrm{C}$.

\section{Heel or finger prick test}

A blood sample will be collected at baseline and at each of the follow-up visit via a heel or finger prick test using a lancet, and a few drops of the blood soaked onto preprinted collection cards (Whatman filter paper). These specimens will be used to measure vitamin $\mathrm{D}$, epigenetics and other biomarkers.

\section{Statistical analysis}

The primary analysis for $\mathrm{AD}$ outcome will be the comparison of primary outcomes between participants in the standard skin care group and the intervention group. The primary analysis will be performed using the modified intention to treat principle, where only participants with data available at 12 months will be included. Multiple imputation will be performed to assess the potential for loss to follow-up to have influenced the study results. For the main dichotomous outcomes ( $\mathrm{AD}$ and food allergy), the magnitude of any between-group differences will be quantified by calculating relative risk ratio, as well as absolute risk, difference and number needed to treat, with 95\% CI for the corresponding population estimates. Further analyses using multivariable log-binomial regression will be undertaken to explore the sensitivity of the effect estimates to imbalance between the groups at baseline on prognostic factors. We will also perform a planned per protocol analysis that only includes those infants whose parents apply the study treatment on at least 5 days per week and excluding control participants whose parents use an emollient as a prophylactic treatment (rather than as a treatment for rash). We will also test if age of commencement of treatment influences the results. To determine if the effect of this treatment depends on inherent predisposition to impaired barrier function, interaction terms will be fitted between 
treatment group and genetic risk factors (including FLG). We will also explore if any preventive effects are mediate either by alteration of skin lipid or microbiome, as there is some evidence that routine emollient use may alter skin biome. $^{32}$

\section{Sample size}

We require 760 participants (380 in each group). With 380 infants per group, we will have, (1) $86 \%$ power to demonstrate that the intervention causes an absolute risk reduction for $\mathrm{AD}$ of $12 \%$ (from $40 \%$ to $28 \%, \mathrm{RR}=0.7$ ), and (2) $80 \%$ power to detect a $7.5 \%$ reduction in food allergy at 12 months (from $15 \%$ to $7.5 \%, \mathrm{RR}=0.5$ ). As the population prevalence of FLG null mutations is approximately $10 \%,{ }^{33}$ and we will only recruit children with a family history of allergic disease, we expect at least $15 \%$ of participants to have one or more FLG null mutation. This will result in 45 infants per group (allowing for up to $80 \%$ loss to follow-up) with one or more such mutations. We will have approximately $80 \%$ power to detect a relative risk of $0.40(20 \%$ vs $50 \%$ ) induced by the treatment for the outcome of $\mathrm{AD}$.

\section{Start of the study}

Recruitment commenced in March 2018.

\section{Patient and public involvement}

Patients and the public were not involved in the development of this study protocol. All participants will be sent an annual study update newsletter and a summary of the study results at the completion of the trial.

\section{Data and safety monitoring}

An independent Data Safety Monitoring Board (DSMB) has been appointed to oversee the safety of the trial, including adverse events monitoring. The DSMB consists of two clinical immunologists/allergists, a biostatistician and dermatologist. Serious safety issues will be reported to the RCH Human Research Ethics Committee. No interim analyses are planned and the DSMB may advise that the study be stopped if they have significant concerns for the safety of study participants. Adverse events, protocol deviations or violations will be documented in REDCap and all serious safety issues reported to the approving ethics committees. A Clinical Trial Monitoring plan has been established with auditing occurring independently of the PEBBLES study team.

\section{DISCUSSION}

This trial will determine whether application of a ceramide-dominant emollient two times per day can prevent the development of $\mathrm{AD}$ (including 6 months after the cessation of treatment) and food allergy. The study findings will help determine if maintenance of the infant skin barrier may help reduce the risk of allergic sensitisation and subsequent allergic diseases. This will demonstrate the role of impaired skin barrier function as an inducer of allergic diseases and may inform primary prevention strategies.
Allergic diseases, including $\mathrm{AD}$, food allergy, allergic rhinitis and allergic asthma are highly prevalent in Westernised countries, and are increasingly common in low and middle income countries. ${ }^{34}$ Collectively, these conditions cause substantial burden of disease. Their rapid increase in prevalence over recent decades ${ }^{35}$ and the substantial differences in their prevalence between countries and cities suggest a key role for environmental exposures. Current therapies for these conditions predominantly aim to minimise symptoms rather than cure (with the possible exception of immunotherapy, ${ }^{36} 37$ which is often a long-term process that is often challenging to implement). While there has been substantial research investment, there remains no proven primary-prevention strategy for these conditions. ${ }^{38} 39$

It is possible that the effect of prophylactic emollient use in infants may be modified by genetic polymorphisms that impair skin barrier function. The primary gene of interest is FLG, which encodes for production of pro-FLG, which is required for the formation of the cornified cell envelope and maintenance of the skin barrier. ${ }^{33}$ Null mutations in the FLG gene are relatively common $(\sim 7 \%-$ $10 \%$ of European populations ${ }^{40}$ ), and there are race-specific FLG null mutations. ${ }^{41}$ These null mutations result in reduced expression of FLG. Null mutations in FLG have been associated with impaired skin barrier function, ${ }^{42}$ $\mathrm{AD}^{29}$ and food sensitisation and allergy. ${ }^{43}$ As mutations in this gene reduce the integrity of the skin barrier, it is plausible that interventions that improve infant skin barrier function may be particularly effective in these individuals. While the evidence supporting the role of FLG null mutations in the aetiology of $\mathrm{AD}$ and food allergy is robust, there are other genes that have also been associated with $\mathrm{AD}$ and impaired skin barrier, including SPINK5. ${ }^{44}$

In this study, we will collect genetic samples from participants at 12 months of age to perform genotyping for polymorphisms in FLG and other genes that are associated with skin barrier impairments, or risk of $\mathrm{AD}$, to determine if the effect of this intervention is modified by these polymorphisms. While the sample size that will be recruited for this study is substantial, we will be relatively underpowered to detect stratum-specific effects (if 10\% of the recruited sample has an FLG null mutation, only 38 participants per group will have such a null mutation). It is likely that results from similar trials will need to be pooled to adequately address this issue.

We have also elected to use the term $\mathrm{AD}$, rather than eczema, as it is a more specific clinical term, ${ }^{45}$ despite eczema being commonly used term in the community. We will use the UK Working Party Criteria for $\mathrm{AD}$ as a primary outcome in this study. ${ }^{23}$ This is a commonly used definition and includes flexural rash that is observed by an assessor blinded to the study group. While this definition does not require evidence of raised specific IgE to diagnose $\mathrm{AD}$, this is appropriate given that $30 \%-40 \%$ of infants with $\mathrm{AD}$ in early life having negative skin prick tests. ${ }^{46}{ }^{47}$ However, we have included IgE-associated AD as a secondary outcome. 
The strengths of the PEBBLES trial include the following: the large sample size, that is targeted to families with a history of allergic disease, with blinded outcome assessment, and including food challenges to confirm food allergy. The study will be conducted in Melbourne, Australia, where there are high rates of $\mathrm{AD}^{48}$ and food allergy. ${ }^{49}$ Inclusion of only infants with a family history of allergic disease will increase the baseline prevalence of $\mathrm{AD}^{48}$ and food allergy, ${ }^{50}$ which will maximise the statistical power to detect an effect of this intervention with the available sample size. Infants with a family history of allergic disease are likely to be motivated to implement such preventive measures if the results show reduction in these outcomes. However, a limitation of this approach is that the results may not be directly generalisable to the whole community or to settings with lower rates of allergic disease.

The selection of a ceramide-dominant emollient is both a strength and limitation of this study. There is evidence to suggest that a ceramide-dominant emollient, with physiological ratios of free fatty acids and cholesterol, is likely to be effective at improving skin barrier function and therefore reduce risk of allergic sensitisation and disease. ${ }^{16}$ As EpiCeram is not available in Australia at this time, there can be no direct contamination of the control group. As we cannot withhold treatments from the control group, there might be use of other emollients for disease prevention, which we will monitor and record. While other ceramide containing treatments are available in Australia, they are much more expensive than standard emollients, limiting their use in the control group. Cost of EpiCeram may impact its potential viability as a population-based intervention.

\section{CONCLUSIONS}

This study is the first to test whether routine prophylactic use of a ceramide-dominant emollient will prevent the development of $\mathrm{AD}$ beyond the active treatment period and prevent the development of food allergy and allergic sensitisation. To date, there are no proven measures that can prevent these common conditions despite substantial research investment. The results of this trial will help clarify the role of the skin barrier as a route of allergic sensitisation leading to subsequent allergic disease. If successful, our trial has the potential to help reduce the burden of $\mathrm{AD}$ and food allergy.

\section{Ethics and dissemination}

Protocol amendments will be submitted to the approving ethics committees and approvals disseminated to the recruitment sites and associated personnel and the PEBBLES investigative team. Any update that impacts participants will necessitate an updated consent which can be actioned in REDCap online (via an email notification) or direct entry at the 6-week or 12-month assessments.

Written informed consent will be obtained from the parent or guardian of all trial participants by study staff.
Consent will be voluntary and free from coercion and participants are free to withdraw at any time without this affecting their future care. The confidentiality of participants will be protected at all times, and no identifying data will be distributed to any third parties. AL and the University of Melbourne Investigators will have full access to the final trial data set.

Trial outcomes will be disseminated through publication and presented at scientific conferences. All study investigators will be given the opportunity to coauthor papers, and the services of professional writers will not be used.

This paper is based on the PEBBLES protocol V.4, 10 July 2018.

\section{Author affiliations}

${ }^{1}$ Allergy and Lung Health Unit, Melbourne School of Population and Global Health,

University of Melbourne, Melbourne, Victoria, Australia

${ }^{2}$ Murdoch Children's Research Institute, Royal Children's Hospital, Parkville, Victoria, Australia

${ }^{3}$ Department of Paediatrics, University of Melbourne, Parkville, Victoria, Australia

${ }^{4}$ Eastern Health, Monash University, Melbourne, Victoria, Australia

${ }^{5}$ Royal Children's Hospital, Parkville, Victoria, Australia

${ }^{6}$ Department of Dermatology, Royal Melbourne Hospital, University of Melbourne, Parkville, Victoria, Australia

${ }^{7}$ Mercy Women's Hospital, Heidelberg, Victoria, Australia

${ }^{8}$ School of Population and Global Health, The University of Western Australia, Perth, Australia

${ }^{9}$ Department of Epidemiology \& Preventive Medicine, School of Public Health \& Preventive Medicine, Monash University, Melbourne, Australia

Acknowledgements We thank Professor Lyle Gurrin for providing statistical guidance and generation of the randomisation sequence.

Contributors $A L$ is the principal investigator and has overall responsibility for all aspects of the PEBBLES trial. He initiated and designed the study with assistance from SD, JS, MT, MA, CFR and NC. SOB is the trial coordinator and responsible for day-to-day participant management and management of ethical approvals. $\mathrm{SH}$ and AS developed the participant recruitment strategy. MM and JH developed the plan for genetic analyses, while CJL developed the protocols and planned analyses for the biological samples (skin biome and breast milk assays). MT and KJA developed the protocol for food allergy outcomes, while JS and GV developed the skin and atopic dermatitis outcome assessments. MA and CFR developed the plans for assessment of respiratory outcomes in future follow-ups. AL and SOB wrote the first draft of this article. All authors were involved in critical revision of the article for important intellectual content. All the authors were involved in final approval of the article. Preparing study design, collection, management, analysis and interpretation of data; writing of the report and the decision to submit the report for publication is the responsibility of $\mathrm{AL}$, the principal investigator.

Funding The PEBBLES study is investigator initiated; the University of Melbourne is the sponsor of the study. This work is supported by an NHMRC project grant (GNT1130010). Primus Pharmaceuticals, the manufacturer of EpiCeram has agreed to donate the intervention emollient to the study free of charge. Primus Pharmaceuticals will have no role in the collection, management, analysis and interpretation of data or decision to publish the study data.

Competing interests MT is a member of the Medical Advisory Board (Oceania) for Nestle Nutrition Institute, a member of the Medical Advisory Board (Australia New Zealand) for Danone Nutricia and a member of the Scientific Advisory Board for Immunology Allergy (Global) for Danone Nutricia; and has received lecture fees from Danone and Nestle Nutrition Institute; and has received travel fees from APAPARI. KJA has received speaker's honoraria from Abbott, Danone, Nestle and Alphapharm. MA has received investigator-initiated grants from Pfizer and Boehringer-Ingelheim for unrelated research. He has also received from Sanofi assistance with conference attendance and an honorarium for an unrelated consultancy.

Patient consent for publication Obtained. 
Ethics approval This study has been approved by the Royal Children's Hospital (RCH) Human Research Ethics Committee (project 37090) and the Mercy Health Human Research Ethics Committee (project 2018-008) and was registered with the Australia and New Zealand Clinical Trials Registry (ACTRN12617001380381) on 28 September 2017 and with Clinical Trials.gov on 9 September 2018 as NCT03667651. The Universal Trial Number for this study is U1111-1201- 0431.

Provenance and peer review Not commissioned; externally peer reviewed.

Open access This is an open access article distributed in accordance with the Creative Commons Attribution Non Commercial (CC BY-NC 4.0) license, which permits others to distribute, remix, adapt, build upon this work non-commercially, and license their derivative works on different terms, provided the original work is properly cited, appropriate credit is given, any changes made indicated, and the use is non-commercial. See: http://creativecommons.org/licenses/by-nc/4.0/.

\section{REFERENCES}

1. lerodiakonou D, Garcia-Larsen V, Logan A, et al. Timing of allergenic food introduction to the infant diet and risk of allergic or autoimmune disease: a systematic review and meta-analysis. JAMA 2016;316:1181-92

2. Martin PE, Eckert JK, Koplin JJ, et al. Which infants with eczema are at risk of food allergy? Results from a population-based cohort. Clin Exp Allergy 2015;45:255-64

3. Lowe AJ, Carlin JB, Bennett CM, et al. Do boys do the atopic march while girls dawdle? J Allergy Clin Immunol 2008;121:1190-5.

4. Lowe AJ, Abramson MJ, Hosking CS, et al. The temporal sequence of allergic sensitization and onset of infantile eczema. Clin Exp Allergy 2007;37:536-42.

5. Hopper JL, Bui QM, Erbas B, et al. Does eczema in infancy cause hay fever, asthma, or both in childhood? Insights from a novel regression model of sibling data. J Allergy Clin Immunol 2012;130:1117-22.

6. Martin PE, Matheson MC, Gurrin L, et al. Childhood eczema and rhinitis predict atopic but not nonatopic adult asthma: a prospective cohort study over 4 decades. J Allergy Clin Immunol 2011;127:1473-9.

7. Burgess JA, Dharmage SC, Byrnes GB, et al. Childhood eczema and asthma incidence and persistence: a cohort study from childhood to middle age. J Allergy Clin Immunol 2008;122:280-5.

8. Khan SJ, Dharmage SC, Matheson MC, et al. Is the atopic march related to confounding by genetics and early-life environment? A systematic review of sibship and twin data. Allergy 2018;73:17-28.

9. Dharmage SC, Lowe AJ, Matheson MC, et al. Atopic dermatitis and the atopic march revisited. Allergy 2014;69:17-27.

10. Nikolovski J, Stamatas GN, Kollias N, et al. Barrier function and water-holding and transport properties of infant stratum corneum are different from adult and continue to develop through the first year of life. J Invest Dermatol 2008;128:1728-36.

11. Choi MJ, Maibach HI. Role of ceramides in barrier function of healthy and diseased skin. Am J Clin Dermatol 2005;6:215-23.

12. Kelleher M, Dunn-Galvin A, Hourihane JO, et al. Skin barrier dysfunction measured by transepidermal water loss at 2 days and 2 months predates and predicts atopic dermatitis at 1 year. $J$ Allergy Clin Immunol 2015;135:930-5.

13. Lowe AJ, Su JC, Allen KJ, et al. A randomized trial of a barrier lipid replacement strategy for the prevention of atopic dermatitis and allergic sensitization: the PEBBLES pilot study. $\mathrm{Br} J$ Dermatol 2018;178:e19-21.

14. Simpson EL, Chalmers JR, Hanifin JM, et al. Emollient enhancement of the skin barrier from birth offers effective atopic dermatitis prevention. J Allergy Clin Immunol 2014;134:818-23.

15. Horimukai K, Morita K, Narita M, et al. Application of moisturizer to neonates prevents development of atopic dermatitis. J Allergy Clin Immunol 2014;134:824-30.

16. Lowe AJ, Leung DYM, Tang MLK, et al. The skin as a target for prevention of the atopic march. Ann Allergy Asthma Immunol 2018;120:145-51.

17. Hoeger PH, Schreiner V, Klaassen IA, et al. Epidermal barrier lipids in human vernix caseosa: corresponding ceramide pattern in vernix and fetal skin. Br J Dermatol 2002;146:194-201.

18. Man MQ M, Feingold KR, Thornfeldt CR, et al. Optimization of physiological lipid mixtures for barrier repair. J Invest Dermatol 1996;106:1096-101.

19. Hachem JP, Crumrine D, Fluhr J, et al. pH directly regulates epidermal permeability barrier homeostasis, and stratum corneum integrity/cohesion. J Invest Dermatol 2003;121:345-53.

20. Sugarman JL, Parish LC. Efficacy of a lipid-based barrier repair formulation in moderate-to-severe pediatric atopic dermatitis. $J$ Drugs Dermatol 2009;8:1106-11.
21. Hengge UR, Ruzicka T, Schwartz RA, et al. Adverse effects of topical glucocorticosteroids. J Am Acad Dermatol 2006;54:1-15. Quiz 6-8.

22. Lowe AJ, Tang ML, Dharmage SC, et al. A phase I study of daily treatment with a ceramide-dominant triple lipid mixture commencing in neonates. BMC Dermatol 2012;12:3.

23. Williams HC, Burney PG, Pembroke AC, et al. The U.K. Working Party's Diagnostic Criteria for Atopic Dermatitis. III. Independent hospital validation. Br J Dermatol 1994;131:406-16.

24. Koplin JJ, Tang ML, Martin PE, et al. Predetermined challenge eligibility and cessation criteria for oral food challenges in the HealthNuts population-based study of infants. J Allergy Clin Immunol 2012;129:1145-7.

25. Hanifin JM, Thurston M, Omoto M, et al. The eczema area and severity index (EASI): assessment of reliability in atopic dermatitis. EASI Evaluator Group. Exp Dermatol 2001;10:11-18.

26. Simpson EL, Keck LE, Chalmers JR, et al. How should an incident case of atopic dermatitis be defined? A systematic review of primary prevention studies. J Allergy Clin Immunol 2012;130:137-44.

27. Asher MI, Keil U, Anderson HR, et al. International Study of Asthma and Allergies in Childhood (ISAAC): rationale and methods. Eur Respir J 1995;8:483-91.

28. Barbier N, Paul C, Luger T, et al. Validation of the Eczema Area and Severity Index for atopic dermatitis in a cohort of 1550 patients from the pimecrolimus cream $1 \%$ randomized controlled clinical trials programme. Br J Dermatol 2004;150:96-102.

29. van den Oord RA, Sheikh A. Filaggrin gene defects and risk of developing allergic sensitisation and allergic disorders: systematic review and meta-analysis. BMJ 2009;339:b2433.

30. Kim J, Kim BE, Lee J, et al. Epidermal thymic stromal lymphopoietin predicts the development of atopic dermatitis during infancy. $J$ Allergy Clin Immunol 2016;137:1282-5.

31. Chng KR, Tay AS, Li C, et al. Whole metagenome profiling reveals skin microbiome-dependent susceptibility to atopic dermatitis flare. Nat Microbiol 2016;1:16106.

32. Glatz M, Jo JH, Kennedy EA, et al. Emollient use alters skin barrier and microbes in infants at risk for developing atopic dermatitis. PLoS One 2018;13:e0192443.

33. Palmer CN, Irvine AD, Terron-Kwiatkowski A, et al. Common loss-of-function variants of the epidermal barrier protein filaggrin are a major predisposing factor for atopic dermatitis. Nat Genet 2006;38:441-6.

34. Pawankar R, Canonica GW, Holgate ST, et al. The WAO White Book on Allergy. 2013

35. Asher MI, Montefort S, Björkstén B, et al. Worldwide time trends in the prevalence of symptoms of asthma, allergic rhinoconjunctivitis, and eczema in childhood: ISAAC Phases One and Three repeat multicountry cross-sectional surveys. Lancet 2006;368:733-43.

36. Abramson MJ, Puy RM, Weiner JM. Injection allergen immunotherapy for asthma. Cochrane Database Syst Rev 2010:CD001186.

37. Tang ML, Ponsonby AL, Orsini F, et al. Administration of a probiotic with peanut oral immunotherapy: a randomized trial. J Allergy Clin Immunol 2015;135:737-44.

38. Foisy M, Boyle RJ, Chalmers JR, et al. Overview of reviews the prevention of eczema in infants and children: an overview of cochrane and non-cochrane reviews. Evid Based Child Health 2011;6:1322-39.

39. Lodge CJ, Allen KJ, Lowe AJ, et al. Overview of evidence in prevention and aetiology of food allergy: a review of systematic reviews. Int J Environ Res Public Health 2013;10:5781-806.

40. Smith FJ, Irvine AD, Terron-Kwiatkowski A, et al. Loss-of-function mutations in the gene encoding filaggrin cause ichthyosis vulgaris. Nat Genet 2006;38:337-42.

41. Chen $\mathrm{H}$, Common JE, Haines RL, et al. Wide spectrum of filaggrinnull mutations in atopic dermatitis highlights differences between Singaporean Chinese and European populations. Br J Dermatol 2011;165:106-14.

42. Flohr C, England K, Radulovic S, et al. Filaggrin loss-of-function mutations are associated with early-onset eczema, eczema severity and transepidermal water loss at 3 months of age. Br J Dermatol 2010;163:1333-6.

43. Tan HT, Ellis JA, Koplin JJ, et al. Filaggrin loss-of-function mutations do not predict food allergy over and above the risk of food sensitization among infants. J Allergy Clin Immunol 2012;130:1211-3.

44. Ashley SE, Tan HT, Vuillermin P, et al. The skin barrier function gene SPINK5 is associated with challenge-proven IgE-mediated food allergy in infants. Allergy 2017;72:1356-64.

45. Silverberg JI, Thyssen JP, Paller AS, et al. What's in a name? Atopic dermatitis or atopic eczema, but not eczema alone. Allergy 2017;72:2026-30. 
46. Lowe AJ, Hosking CS, Bennett CM, et al. Skin prick test can identify eczematous infants at risk of asthma and allergic rhinitis. Clin Exp Allergy 2007;37:1624-31.

47. Kusel MM, Holt PG, de Klerk N, et al. Support for 2 variants of eczema. J Allergy Clin Immunol 2005;116:1067-72.

48. Martin PE, Koplin JJ, Eckert JK, et al. The prevalence and sociodemographic risk factors of clinical eczema in infancy: a populationbased observational study. Clin Exp Allergy 2013;43:642-51.
49. Osborne NJ, Koplin JJ, Martin PE, et al. Prevalence of challengeproven IgE-mediated food allergy using population-based sampling and predetermined challenge criteria in infants. J Allergy Clin Immunol 2011;127:668-76.

50. Koplin JJ, Allen KJ, Gurrin LC, et al. The impact of family history of allergy on risk of food allergy: a population-based study of infants. Int J Environ Res Public Health 2013;10:5364-77. 